\title{
A novel workflow combining plaque imaging, plaque and plasma proteomics identifies biomarkers of human coronary atherosclerotic plaque disruption
}

Regent Lee 1,3,4, Roman Fischer ${ }^{3}$, Philip D. Charles ${ }^{3}$, David Adlam ${ }^{1}$, Alessandro Valli ${ }^{3}$, Katalin Di Gleria ${ }^{3}$, Rajesh K. Kharbanda ${ }^{1,4}$, Robin P. Choudhury ${ }^{1,2}$, Charalambos Antoniades ${ }^{1,4}$, Benedikt M. Kessler ${ }^{3}$ and Keith M. Channon ${ }^{1,4^{*}}$

\begin{abstract}
Background: Atherosclerotic plaque rupture is the culprit event which underpins most acute vascular syndromes such as acute myocardial infarction. Novel biomarkers of plaque rupture could improve biological understanding and clinical management of patients presenting with possible acute vascular syndromes but such biomarker(s) remain elusive. Investigation of biomarkers in the context of de novo plaque rupture in humans is confounded by the inability to attribute the plaque rupture as the source of biomarker release, as plaque ruptures are typically associated with prompt down-stream events of myocardial necrosis and systemic inflammation.

Methods: We developed a novel approach to identify potential biomarkers of plaque rupture by integrating plaque imaging, using optical coherence tomography, with both plaque and plasma proteomic analysis in a human model of angioplasty-induced plaque disruption.

Results: We compared two pairs of coronary plaque debris, captured by a FilterWire Device, and their corresponding control samples and found matrix metalloproteinase 9 (MMP9) to be significantly enriched in plaque. Plaque contents, as defined by optical coherence tomography, affect the systemic changes of MMP9. Disruption of lipid-rich plaque led to prompt elevation of plasma MMP9, whereas disruption of non-lipid-rich plaque resulted in delayed elevation of plasma MMP9. Systemic MMP9 elevation is independent of the associated myocardial necrosis and systemic inflammation (measured by Troponin I and C-reactive protein, respectively). This information guided the selection of a subset of subjects of for further label free proteomics analysis by liquid chromatography tandem mass spectrometry (LC-MS/ MS). We discovered five novel, plaque-enriched proteins (lipopolysaccharide binding protein, Annexin A5, eukaryotic translocation initiation factor, syntaxin 11, cytochrome B5 reductase 3) to be significantly elevated in systemic circulation at 5 min after plaque disruption.
\end{abstract}

Conclusion: This novel approach for biomarker discovery in human coronary artery plaque disruption can identify new biomarkers related to human coronary artery plaque composition and disruption.

Keywords: Coronary atherosclerosis, Plaque rupture, Proteomics, Biomarkers

\footnotetext{
*Correspondence: keith.channon@cardiov.ox.ac.uk

1 Division of Cardiovascular Medicine, University of Oxford, Oxford, UK

Full list of author information is available at the end of the article
} 


\section{Background}

Atherosclerotic plaque rupture is the culprit event which underpins a majority of acute vascular syndromes such as acute myocardial infarction [1], yet biomarkers of plaque rupture remain elusive $[2,3]$. Novel biomarkers of plaque rupture could improve clinical management of patients presenting with possible acute vascular syndromes, and give new insights to the pathophysiological process causing plaque rupture, and the local and systemic consequences.

One of the challenges in the investigations of plaque rupture in humans lies in the difficulty to establish the time course of a de novo plaque rupture event. In this regard, plaque disruption by percutaneous coronary intervention (PCI) induces controlled plaque rupture in humans and allows profiling of biomarker changes timed precisely to a plaque event [4-6]. Furthermore, intraarterial imaging of plaque in vivo using optical coherence tomography (OCT) can define plaque composition, providing additional information to improve the specificity of biomarker discovery [6-9].

However, even in such controlled settings, characterisation of biomarker of plaque disruption are further complicated by the presence of downstream effects such as myocardial necrosis and systemic inflammation after PCI $[7,10,11]$, as these downstream events also lead to changes in cardiac enzymes (cTnI) and c-reactive protein (CRP) and can confound the identification of other circulating biomarkers that are specifically associated with plaque disruption.

In this study, we applied a novel discovery workflow to the human model of PCI-induced plaque disruption. We hypothesised that by combining the information from coronary plaque imaging analysis, proteomics analysis of coronary plaque material, and proteomics analyses of serial plasma samples collected before and after plaque disruption, this would enable identification plaque proteins acutely elevated in systemic circulation that are specific markers of human coronary artery plaque disruption.

\section{Methods \\ Model of human plaque disruption in vivo}

In a prospective study at the Oxford Radcliffe Hospital (ORH), Oxford, UK, patients between the age of 30-90 who were referred for non-emergency coronary angiography with the view to proceed to PCI were invited to take part. Patients who underwent diagnostic angiography without stenting served as control subjects in this experimental model as the steps involved in diagnostic angiography (arterial needle puncture/catheterisation, intra-arterial passage of guidewires, injection of contrast and acquisition of X-ray images) were the same as PCI except deployment of stents. We excluded patients who were haemodynamically unstable, or when there was a known history of renal impairment or anaemia. Patients undergoing left main coronary artery angioplasty were also excluded. The study was approved by the Oxford Regional Ethics Committee (Ethics Ref: 08/H0603/41) and complies with the Helsinki Declaration. Written informed consent was obtained from every participant.

\section{Characterisation of plaque morphology by optical coherence tomography (OCT)}

OCT images were acquired using the Lightlab system (St. Jude Medical, Minnesota, USA) prior to disruption of plaque by stent. Image analyses were performed by assessors who were blinded to the clinical status and other biomarker measurements of the patient. The culprit lesion was categorised into two subtypes (lipid-rich and non-lipid rich) using the pre-defined scoring system. (Full details on OCT image acquisition and analysis please in Additional file 1.) Discordance between observers were resolved by taking a consensus reading. A binary score was then assigned to each culprit lesion for comparison with other variables.

\section{Sampling of coronary plaque material}

To obtain coronary plaque material released after its disruption, a FilterWire device (FW, Boston Scientific, Massachusetts, USA) was deployed downstream of the lesion in a standard manner. The umbrella-like polyurethane mesh contains $110 \mu \mathrm{m}$ pores to allow passage of blood components while trapping the plaque material released during angioplasty. To assess if blood components (other than coronary plaque material) would also adhere to the FW during the PCI procedure, therefore giving rise to artefactual signals unrelated to coronary plaque material, a paired FW device was prepared concurrently as the matched control sample in the following manner: after the diagnostic angiography confirmed suitability for stenting with FW distal protection, an additional $10 \mathrm{ml}$ of arterial blood was aspirated from the coronary guide catheter and transferred into a $15 \mathrm{ml}$ Falcon tube. A FW was then positioned downstream of the coronary lesion followed by the PCI procedure. Concurrently, an unused FW was placed in the Falcon tube containing the individual's blood. This was then immediately transferred to a water bath and incubated at $37.5{ }^{\circ} \mathrm{C}$ for the duration of the stenting procedure (Fig. 1). At the end of the PCI procedure, both FW (one containing plaque material, and one from ex vivo incubation) were retrieved and immediately frozen and stored at $-80^{\circ} \mathrm{C}$ for subsequent analysis.

\section{Serial plasma sampling}

Blood samples were collected from coronary artery and peripheral vein at specific time points after baseline. 
a

\section{Collection of plaque material sample and control}
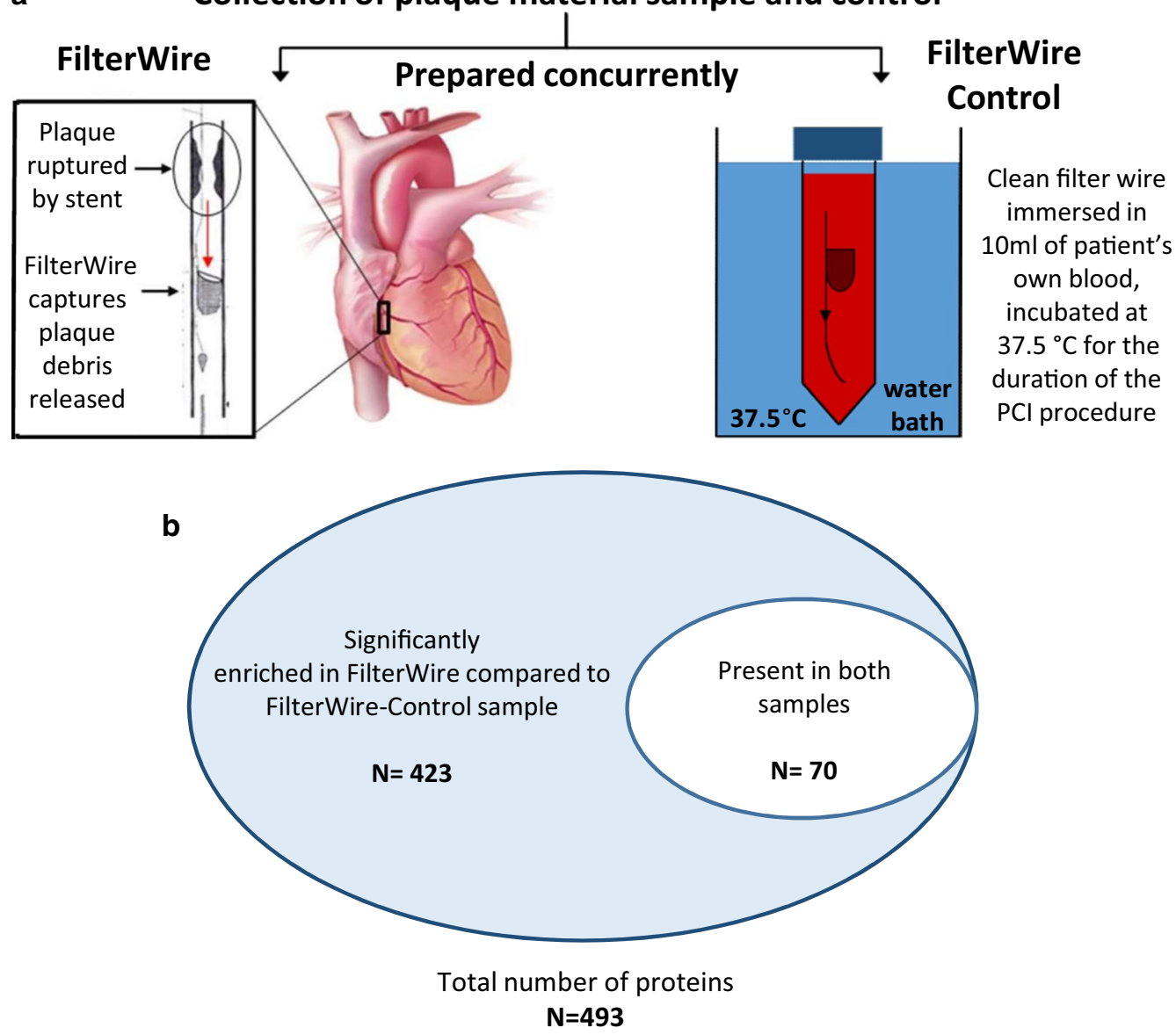

Fig. 1 Establishing a library of protein that are present in coronary plaque debris, captured by the FilterWire device. a In order to identify proteins acutely released into circulation from a disrupted plaque, we first established a library of plaque proteins by untargeted proteomic analysis of plaque debris captured by a distal protection device with pore size of $110 \mu \mathrm{m}$. (FilterWire, Boston Scientific), positioned in the coronary artery downstream from the plaque during PCl. Concurrently, a second FilterWire was incubated at $37.5^{\circ} \mathrm{C}$ ex vivo in heparinised blood from the same patient (obtained at the beginning of the procedure, before angioplasty), for the same duration as the PCI procedure. This separate FW served as a control sample for the one deployed in the coronary artery in the same patient, in order to identify the non-specific blood proteins which may adhere to the FW during the $\mathrm{PCl}$ process, other than plaque debris. $\mathbf{b}$ These paired samples were analysed using the proteomics workflow as described in the manuscript. 423 proteins were significantly enriched in FilterWire compared to FilterWire-Control and considered to be plaque specific proteins

Standard guiding catheters were used for blood sampling from proximal coronary artery during the PCI procedure (at baseline and at $5 \mathrm{~min}$ ). Peripheral venous blood samples were obtained from either indwelling venous catheters or fresh venepuncture (at baseline, 1, 6, $18 \mathrm{~h}$ ). The same time points applied to diagnostic angiography patients except for stent placement, and the beginning of procedure was used as the baseline. Blood samples were prepared without delay in the cardiac catheter laboratory at room temperature to obtain platelet-poor plasma with the following protocol: BD Vacutainer ${ }^{\circledR}$ containing $\mathrm{K}_{2}$ EDTA was used for the first spin of blood $(12 \mathrm{~min} \times 1300 \mathrm{~g})$. The plasma portion was then transferred to a second BD Vacutainer ${ }^{\circledR}$ without additive for the second spin $(15 \mathrm{~min} \times 2500 \mathrm{~g})$. Confirmation of platelet-poor plasma was confirmed by platelet counts [12]. Plasma aliquots were stored at $-80{ }^{\circ} \mathrm{C}$ for subsequent analysis.

\section{Candidate marker analysis}

Measurement of plasma CRP, cTnI (both by high sensitivity assays), and blood cholesterol profiles were performed as clinical blood tests by the biochemistry at the Oxford Radcliffe Hospital. Other candidate markers were measured using commercially available bead based immune-sorbent assays according to the manufacturer's instructions. (MMP9: HCVD1-67AK, Merck Millipore, USA; LBP: Mybiosource, MBS721952, USA). Intra- and 
inter-plate variations (\%CV) for these assays were less than $10 \%$.

\section{Statistical analysis for candidate markers}

Dependent on the normality of sample distribution, comparisons between two groups were performed by either parametric or non-parametric t-tests. Log transformation of data was performed where appropriate. Analysis of variance (ANOVA) was performed for comparison between 3 or more groups or to test the effects of repeated measures. Area under curve (AUC) of the time course response for each candidate marker was calculated. These analyses were performed using Graphpad Prism (Version 5.0, San Diego California USA).

Data with a Gaussian distribution are presented as mean \pm standard error of mean; parametric data are presented as median and $25-75$ percentile in box plots. Area under curve (AUC) can be seen as relative measure of abundance in release over a period, which allows comparisons across samples/patients. AUC over the time course of each biomarker was calculated using the "area under curve" analysis in GraphPad Prism. As there is a broad physiological range of baseline MMP9 concentration between individuals, the fold change of MMP9 at each time point (against baseline) was used for the AUC calculation. In contrast, the baseline levels of CRP and Troponin are fairly consistent between individuals (hence their role as diagnostic tests). The AUC for CRP and Troponin are therefore calculated using the absolute concentration measured at each time point.

Correlation between MMP9 and known injury and inflammation response biomarkers (Troponin and CRP) was assessed by Spearman's rank correlation coefficient (Spearman's rho) across individuals to capture any monotonic relationship (linear or otherwise). Significance was determined by converting the Fisher transformation of the rho values to $\mathrm{z}$-scores, thus yielding corresponding $P$ values by assuming the $\mathrm{z}$-scores to follow a standard normal distribution (calculations performed in R).

\section{Sample size consideration for proteomics analysis}

Sample size consideration in comparative proteomics research, whereby samples are compared according to a disease condition (such as before and after plaque disruption) to screen for up or down regulation of "novel/ unknown" molecular signatures, is different from the traditional power calculation approach for "known" candidate markers [13]. Assuming the combined biological and technical variance of $75 \%$ between samples, the sample size of 10 in each arm of the study will be sufficient to detect a 1.9 fold change (up or down regulation) in a given protein/metabolite with $80 \%$ power and $\alpha$ of 0.05 [14].

\section{Untargeted proteomic analysis of coronary plaque and plasma samples}

For the discovery analysis by shot gun proteomics, we compared plasma samples taken at baseline and at $5 \mathrm{~min}$ to capture the earliest changes detectable in circulation. Changes observed at this early time point are most likely due to acute liberation of pre-existing proteins into circulation. Plasma samples for each group were pooled for subsequent analysis, as pooling gave the advantage of reducing the intrinsic biological variation observed between samples, making the substantive features easier to detect, and reduced the total number of samples analysed and reduce the overall requirement of computational power for the analysis and the constraints imposed by instrument throughput and cost considerations $[15,16]$. Samples were prepared using in-solution digestion with trypsin. Mass spectra of these samples were acquired using LC-MS/MS (LTQ-orbitrap Velos, Thermo Fisher Scientific). For detailed description of the sample preparation and mass spectra acquisition, please see Additional file 1.

\section{Label-free relative quantitation of proteins}

For label-free protein quantitation, raw data were first imported to Progenesis LC-MS and processed through the default workflow. Proteins were grouped and only protein features which were identified by two or more tryptic peptides were included for identification. Statistical comparisons were made in the following experimental groups: FW versus FW-Controls, plasma no plaque disruption ( 0 vs. $5 \mathrm{~min}$ ), plasma plaque disruption ( 0 vs. $5 \mathrm{~min}$ ). For the comparison between FW and FW-controls, normalisation of protein abundance was not performed because of the vastly low abundance of protein in the FW-Controls. For the comparison between paired plasma samples, normalisation was performed against all peptides as there were comparable intensity of mass spectra in paired samples. $P$ values for comparison were calculated by the Progenesis LC-MS software, based on paired $t$ tests (two sample ANOVA), and the value of $<0.05$ was considered the cut-off point for selection of a putative protein feature for further examination.

Only peptides identified with a false discovery rate of $1 \%$ and with ion score of $>20$ were accepted for further analysis. False discovery rate (FDR) of the protein identification was further performed by uploading the converted MGF files to the Central Proteomics Facilities Pipeline (CPFP, version 1.3.0 http://www.proteomics.ox.ac.uk/), which combined the searches against the human databases using three different search engines (Mascot, OMSSA, and X!TANDEM k-score) [17]. The FDR for each protein was cross referenced between CPFP output file and Progenesis LC-MS data. Of these proteins 
which had a $P$ value less than 0.05 on semi-quantitative analysis, only those which fulfilled a FDR of less than $1 \%$ according to CPFP analysis were considered for further analysis.

\section{Ingenuity Pathway Analysis (IPA) of proteomics datasets}

The complete plasma protein dataset for the individual experimental groups were further exported from Progenesis LC-MS. For each dataset, the following values were assigned as an observation in IPA: protein accession number, ANOVA $P$ value, fold change at 5 min (compared to $0 \mathrm{~min}$ ). Canonical pathways implicated by the plasma proteins in the sample groups were assessed using the over-representation technique in IPA. This analysis provided the significance $P$ value based on likelihood of representation and the percentage representation of proteins detected in the dataset. Benjamini-Hochberg correction for multiple testing was applied to the $P$ values.

\section{Results}

We imaged coronary artery plaques, using optical coherence tomography (OCT) prior to plaque disruption, in 36 patients undergoing percutaneous coronary intervention (PCI). These plaques were classified as either lipidrich $(\mathrm{n}=23)$ or non-lipid-rich $(\mathrm{n}=13)$ plaques based on OCT characteristics. Lipid-rich and non-lipid-rich plaques were observed in patients who presented with either acute coronary syndrome (ACS) or stable angina (SA) (Fig. 2a).

We first analysed plaque debris collected "downstream" from the coronary artery plaque during PCI, using a FilterWire device. The FW and the FW-Control were processed concurrently for proteomics analyses. We identified a total of 493 proteins from these FW and FW-control samples. Of these, 423 proteins were derived from the plaque debris as they were significantly enriched $(P<0.05)$ in the FW samples as compared to the paired FW-Control (Fig. 1). For the complete list please see Additional file 2. Matrix metalloproteinase 9 (MMP9) was one of the proteins significantly enriched in the plaque debris. Given the known involvement of MMP9 in vulnerable plaques [18], we selected MMP9 as a surrogate marker of the response to human coronary artery plaque disruption.

To test whether putative biomarkers of human coronary plaque disruption are also detectable in the peripheral circulation, we next measured plasma MMP9 levels after plaque disruption, in relation to clinical status [ACS $(\mathrm{n}=21)$ vs. SA $(\mathrm{n}=37)]$ and plaque morphology [lipidrich $(\mathrm{n}=23)$ vs. non-lipid-rich $(\mathrm{n}=13)]$. More prompt elevation in plasma MMP9 was observed in patients who presented with ACS (fold change 1.37 $\pm 0.08, P<0.05$ against baseline) at $1 \mathrm{~h}$ after plaque disruption (as compared to those who presented to SA with fold change of $1.16 \pm 0.06)$. By $18 \mathrm{~h}$ after plaque disruption, both ACS and SA patients showed similar fold changes (ACS: $1.98 \pm 0.46$; SA: $1.79 \pm 0.16$ ). In contrast, disruption of lipid-rich plaques led to more prompt elevations of MMP9 (fold change $1.28 \pm 0.07$ at $1 \mathrm{~h}, P<0.05$ against baseline) and higher peak level after $6 \mathrm{~h}$ (fold change $1.96 \pm 0.16)$ as compared to non-lipid-rich plaques (fold change $1.1 \pm 0.08$ at $1 \mathrm{~h}$, and $1.44 \pm 0.15$ at $6 \mathrm{~h}$ ) (Fig. 2b). There was no gender difference in the MMP9 levels observed (Data not shown). Taken together, these observations suggest plaque composition determines biomarker release after plaque disruption, providing the basis for novel biomarker discovery associated with plaque contents.

To test for possible confounding effects of myocardial injury and systemic inflammatory response after plaque disruption, we measured circulating troponin I (TnI) and C-reactive protein (CRP) in all participants $(\mathrm{PCI} n=58$; Dx $\mathrm{n}=23$, Table 1). Myocardial injury was observed only in the group with plaque disruption, after $6 \mathrm{~h}$. Modest

(See figure on next page.)

Fig. 2 Plaque composition affects systemic elevation of MMP9 after its disruption, which is independent of the myocardial injury and systemic inflammatory response. Coronary artery plaques were imaged using optical coherence tomography (OCT) prior to plaque disruption, in 36 patients undergoing percutaneous coronary intervention ( $\mathrm{PCl}$ ), comparing with subjects who underwent diagnostic coronary angiography without $\mathrm{PCl}(\mathrm{Dx})$. These plaques were classified as either lipid-rich (yellow, $n=23$ ) or non-lipid-rich plaques (green, $n=13$ ) based on OCT characteristics. Lipid-rich and non-lipid rich plaques were observed in patients with either acute coronary syndrome (ACS-red, $n=21)$ or stable angina (SA-blue, $n=37)(\mathbf{a})$. More prompt elevation in plasma MMP9 was observed in patients who presented with ACS (b, red bars) at $1 \mathrm{~h}$ after plaque disruption (compared to those who presented to SA, blue bars), but there was no difference in the peak MMP9 levels observed after $6 \mathrm{~h}$. In contrast, disruption of lipidrich plaques (b, yellow bars) led to more prompt elevations of MMP9 and higher peak level after $6 \mathrm{~h}$. To test for possible confounding effects of myocardial injury and systemic inflammatory response after plaque disruption, we measured circulating troponin I (Tnl) and c-reactive protein (CRP) in all participants $(\mathrm{PCl} n=58 ; \mathrm{Dx} n=23)$. Myocardial injury was only observed in the group with plaque disruption. Modest elevation in CRP was observed in both groups, with or without plaque disruption (c). Calculation of area under curve (AUC) revealed no correlation between MMP9 and either Tnl or CRP (d) during this time course. These observations indicate that MMP9 release is specific to the upstream event of plaque disruption, and independent of the downstream myocardial injury and systemic inflammation as a result of the procedure. "* denotes significant statistical comparison against the baseline measurement (paired comparison); "\#” denotes significant statistical comparison between the two groups (lipidrich vs. non-lipid-rich) 

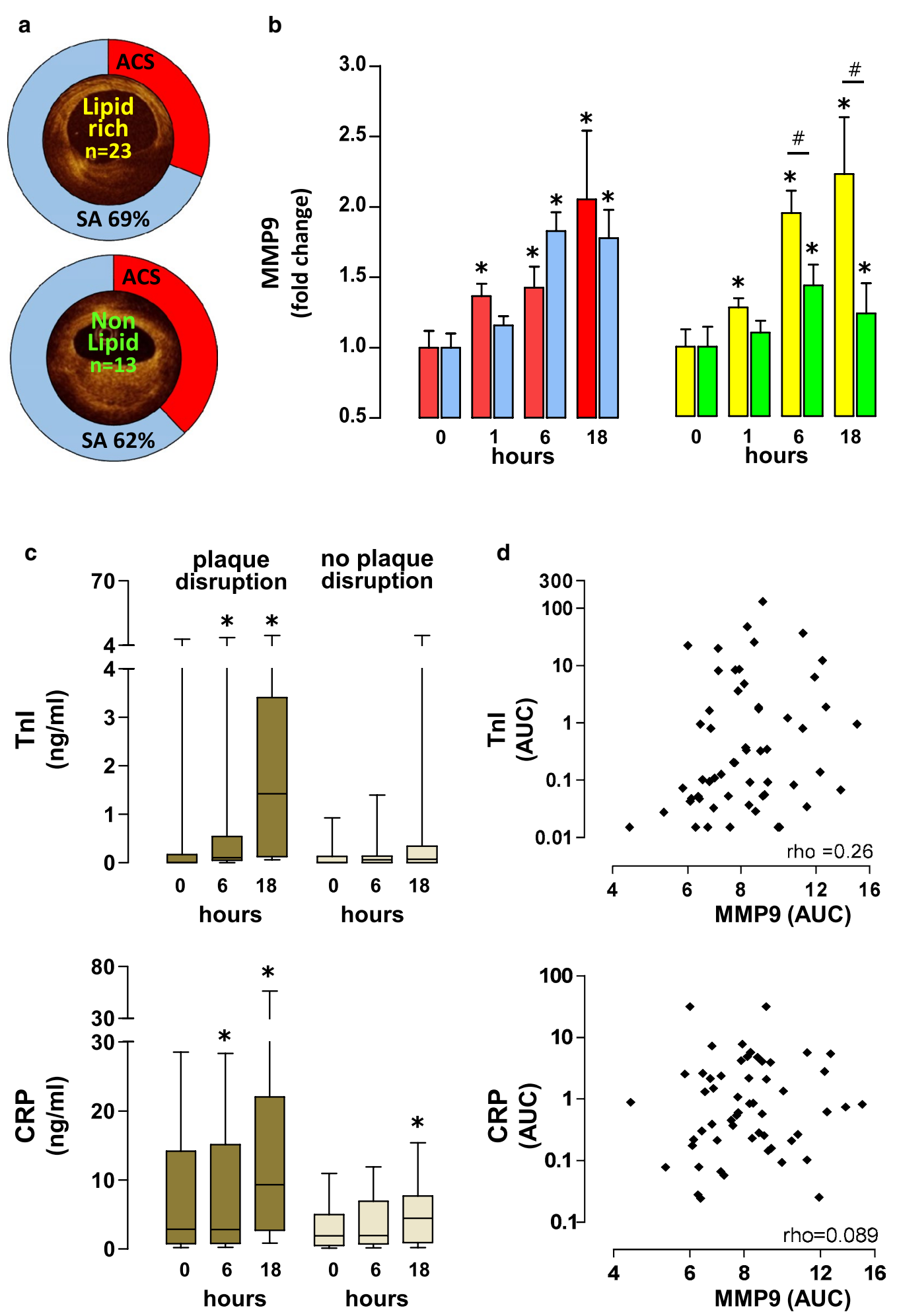
Table 1 Characteristics of study subjects included for overall analysis

\begin{tabular}{lcc}
\hline & PCI & $\begin{array}{l}\text { Diagnostic } \\
\text { angiography }\end{array}$ \\
\hline Number (male) & $58(46)$ & $23(16)$ \\
Age [years (SD)] & $66(11)$ & $68(10)$ \\
Acute coronary syndrome [n (\%)] & $21(36)$ & $13(57)$ \\
Smoking status [n (\%)] & & \\
$\quad$ Current smoker & $16(28)$ & $3(13)$ \\
Ex-smoker >1 month & $25(43)$ & $13(57)$ \\
Never smoked & $17(29)$ & $7(30)$ \\
Past history of IHD [n (\%)] & & \\
Ml/ACS & $22(38)$ & $7(30)$ \\
Stable angina & $15(26)$ & $7(30)$ \\
Hypertension [n (\%)] & $39(67)$ & $19(83)$ \\
Hypercholesterolemia [n (\%)] & $45(78)$ & $13(57)$ \\
Diabetes mellitus [n (\%)] & $14(24)$ & $4(17)$ \\
Family history of IHD [n (\%)] & $29(50)$ & $12(52)$ \\
Regular medication [n (\%)] & & \\
Aspirin & $40(69)$ & $17(74)$ \\
Thienopyridine & $23(40)$ & $11(48)$ \\
Statin & $47(81)$ & $20(87)$ \\
B-blocker & $33(57)$ & $14(61)$ \\
ACE inhibitor/ARB & $38(65)$ & $19(83)$ \\
\hline
\end{tabular}

elevation in CRP was observed in both groups, with or without plaque disruption (Fig. 2c). Quantification of area under curve (AUC) values were consistent with a null hypothesis of no correlation between MMP9 and either TnI $(P=0.91)$ or CRP $(P=\sim 1)$ during this time course (Fig. 2d). These observations indicate that MMP9 release is specific to the upstream event of plaque disruption, and independent of the downstream myocardial injury and systemic inflammation as a result of the procedure.

To characterise 'upstream' biomarkers released immediately after plaque disruption, we used systemic MMP9 levels as a stratification tool to select a discovery cohort of patients for proteomics analyses of plasma samples collected at $5 \mathrm{~min}$ after plaque disruption. This cohort consisted of subjects with the highest MMP9 release (AUC) after plaque disruption, compared with a control group who underwent diagnostic angiography only. In these PCI patients with the highest $\mathrm{MMP}_{\mathrm{AUC}}$, we observed a median fold change of 1.9 at $6 \mathrm{~h}$ and 2.3 at $18 \mathrm{~h}$. Subjects were well matched in their baseline demographics (Table 2).

We detected 491 proteins in these plasma samples with a false discovery rate less than $1 \%$. Canonical pathway analysis, using Ingenuity Pathway Analysis (IPA), highlighted the key pathways represented by these plasma
Table 2 Demographics for the discovery cohort for proteomics analysis

\begin{tabular}{|c|c|c|}
\hline & $\begin{array}{l}\text { Plaque } \\
\text { disruption }\end{array}$ & $\begin{array}{l}\text { No plaque } \\
\text { disruption }\end{array}$ \\
\hline Number (male) & $10(7)$ & $10(5)$ \\
\hline Age [years (SD)] & $66(8)$ & $67(12)$ \\
\hline Acute coronary syndrome [n (\%)] & $5(50)$ & $6(60)$ \\
\hline \multicolumn{3}{|l|}{ Smoking status [n (\%)] } \\
\hline Current smoker & $1(10)$ & $1(10)$ \\
\hline Past history of smoking (>1 month) & $7(70)$ & $4(40)$ \\
\hline Never smoked & $2(20)$ & $5(50)$ \\
\hline \multicolumn{3}{|l|}{ Past History of IHD [n (\%)] } \\
\hline MI/ACS & $2(20)$ & $5(50)$ \\
\hline Stable angina & $4(40)$ & $0(0)$ \\
\hline Hypertension [n (\%)] & $6(60)$ & $8(80)$ \\
\hline Hypercholesterolemia [n (\%)] & $8(80)$ & $6(60)$ \\
\hline Diabetes mellitus [n (\%)] & $0(0)$ & $0(0)$ \\
\hline Family history of IHD [n (\%)] & $6(60)$ & $5(50)$ \\
\hline \multicolumn{3}{|l|}{ Regular medication [n (\%)] } \\
\hline Aspirin & $6(60)$ & $8(80)$ \\
\hline Thienopyridine & $3(30)$ & $4(30)$ \\
\hline Statin & $7(70)$ & $9(70)$ \\
\hline$\beta$-blocker & $5(50)$ & $6(50)$ \\
\hline ACE inhibitor/ARB & $6(60)$ & $7(60)$ \\
\hline \multicolumn{3}{|l|}{ Plasma lipid profile } \\
\hline Total cholesterol [mmol/L (SD)] & $3.7(0.4)$ & $3.8(1.39)$ \\
\hline $\mathrm{HDL}[\mathrm{mmol} / \mathrm{L}(\mathrm{SD})]$ & $1.0(0.3)$ & $0.9(0.3)$ \\
\hline $\mathrm{LDL}[\mathrm{mmol} / \mathrm{L}(\mathrm{SD})]$ & $2.0(0.3)$ & $2.3(1.0)$ \\
\hline Triglyceride [mmol/L (SD)] & $1.5(0.7)$ & $1.5(0.6)$ \\
\hline
\end{tabular}

proteins, with the highest representation being the Liver $\mathrm{X}$ receptor (LXR)/retinoic X receptor (RXR) pathway (Fig. 3). After exclusion of plasma proteins that were significantly changed in the control group (those who underwent diagnostic angiography only), we identified six plasma proteins that were significantly elevated $(P<0.05)$ at $5 \mathrm{~min}$ after plaque disruption. These were compared against the plaque protein library determined from plaque debris. Five of these proteins were also enriched in plaque debris (Table 3 ).

Lipopolysaccharide binding protein (LBP) is one of the protein that is present in human coronary artery plaque and is significantly elevated in plasma immediately after plaque disruption. Elevation of plasma LBP levels after PCI (from $0.62 \pm 0.49$ to $0.74 \pm 0.33 \mathrm{ng} / \mathrm{ml}, 1.44$ times increase [ $95 \%$ confidence interval of $1.07-1.81]$ ) was validated by an enzyme immunoassay in individual plasma samples, but remained unchanged in the control group who underwent diagnostic angiography without plaque disruption (Additional File 3). The significant elevation of LBP was no longer observed beyond $1 \mathrm{~h}$ after plaque 


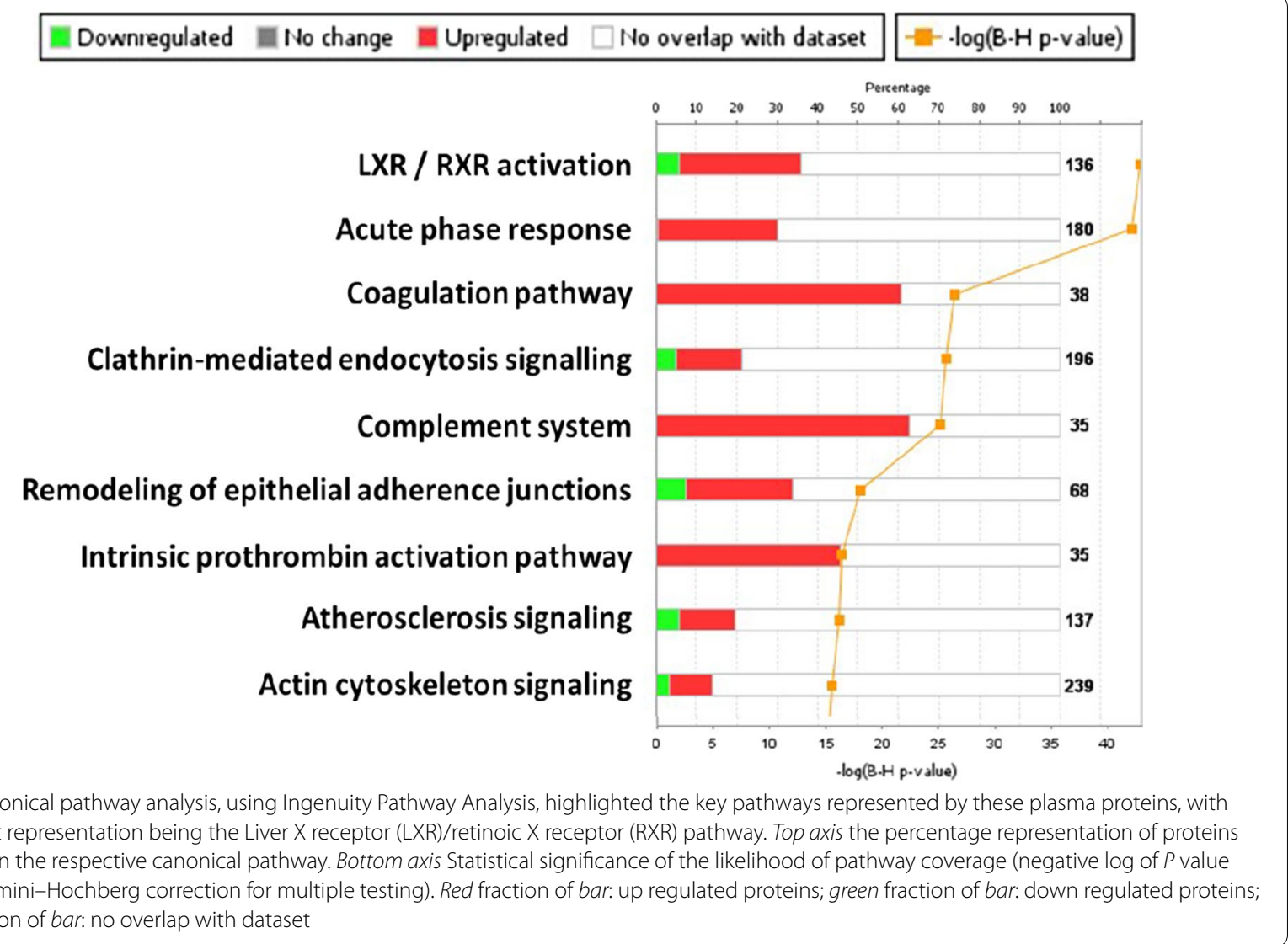

Table 3 Proteins that are significantly increased in systemic circulation 5 min after plaque disruption

\begin{tabular}{|c|c|c|c|c|}
\hline Uniprot accession number & Protein name & Maximum fold change & ANOVA $P$ value & Enriched in plaque \\
\hline P08758 & Annexin A5 & 2.41 & 0.0143 & Yes \\
\hline P18428 & Lipopolysaccharide binding protein & 1.75 & 0.0159 & Yes \\
\hline P63241 & Eukaryotic translation initiation factors & 2.28 & 0.0218 & Yes \\
\hline O75558 & Syntaxin 11 & 3.04 & 0.0222 & Yes \\
\hline P00387 & Cytochrome B5 reductase 3 & 3.88 & 0.0401 & Yes \\
\hline Q9UIB8 & CD84 & 7.18 & 0.0486 & No \\
\hline
\end{tabular}

We detected 491 proteins in these plasma samples with a false discovery rate of less than $1 \%$. After exclusion of plasma proteins that were significantly changed in the control group (those who underwent diagnostic angiography only), we identified six plasma proteins that were significantly elevated $(P<0.05)$ at 5 min after plaque disruption. These were cross referenced against the plaque protein library determined from plaque debris. Five of these proteins were also present in plaque debris

disruption, and there was no correlation between $\mathrm{LBP}_{\mathrm{AUC}}$ and $\mathrm{TnI}_{\mathrm{AUC}}$ or between $\mathrm{LBP}_{\mathrm{AUC}}$ and $\mathrm{CRP}_{\mathrm{AUC}}$ (data not shown).

This novel workflow is illustrated by Fig. 4 .

\section{Discussion}

We describe a novel approach for biomarker discovery in a human model of PCI-induced coronary atherosclerotic plaque disruption. We combined plaque OCT imaging to stratify plaque composition, with discovery proteomics of both plaque debris and plasma samples, to identify and validate proteins released from human coronary artery plaques immediately after plaque disruption. This is the first time a study took such a rigorous approach of using plaque analysis to guide subsequent proteomics discovery in plasma, illustrating the potential of this approach to identify new targets and markers related to human coronary artery plaque biology.

We used OCT as a means of increasing the specificity of the study to detect proteins related to disruption 


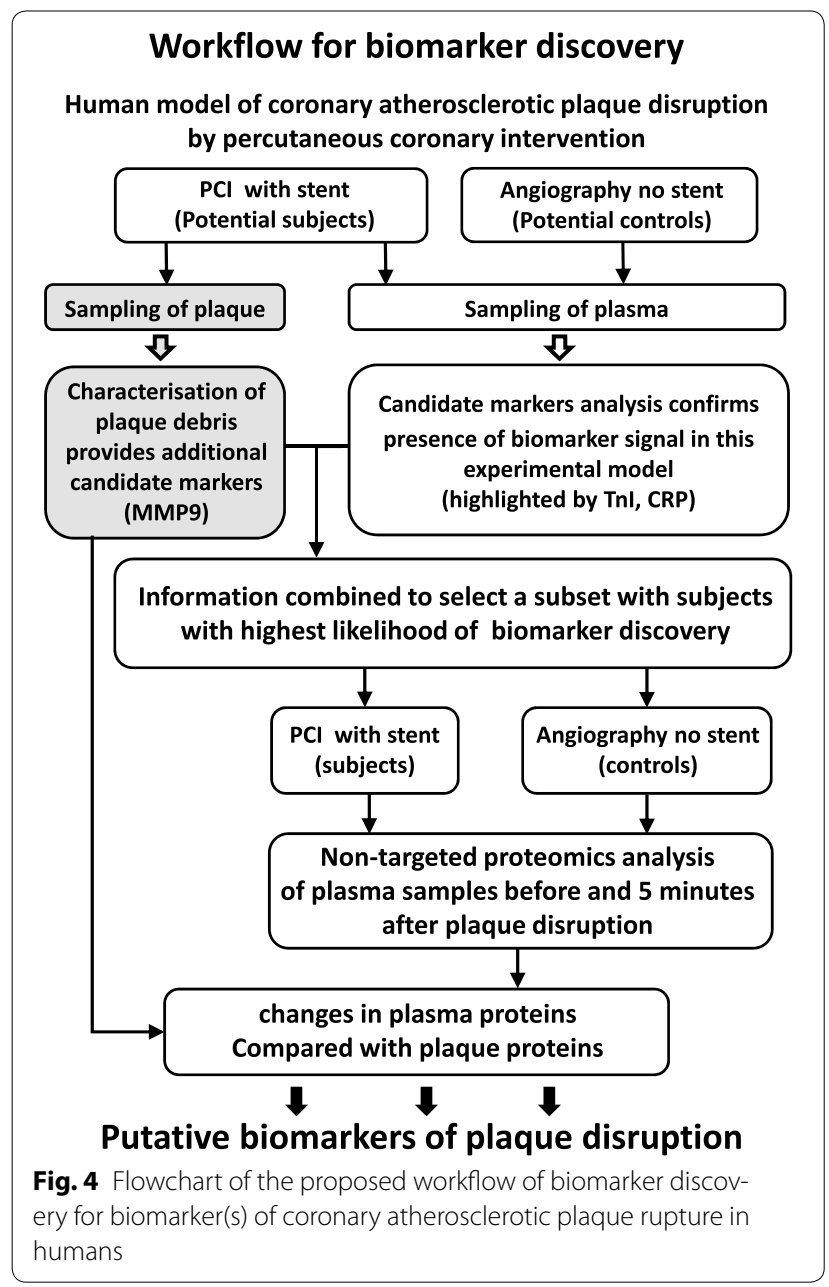

of lipid-rich plaques, because these plaques ('unstable' or 'vulnerable' plaques) are the substrates for spontaneous plaque rupture that leads to acute coronary events. We observed that the pattern of biomarker release after plaque disruption (exemplified by MMP9) was independent of the clinical presentation of the patient (i.e. ACS vs. SA), but was significantly different between lipid-rich plaques compared with non-lipid plaques, demonstrating that plaque composition directly influences biomarker release after plaque disruption.

Our finding of systemic elevation in MMP9 within an hour after coronary plaque disruption is consistent with its reported role in atherosclerotic plaque destabilisation $[19,20]$. That systemic MMP9 elevation after plaque disruption did not correlate with cTnI or CRP release further supports MMP9 release to be an independent feature specific to the plaque disruption.

The potential discovery utility of the integrated plaque imaging proteomic analysis approach highlights the power of combining proteomic analysis of both plaque-derived particulate debris and plasma proteins, in a rigorous and highly controlled experimental design. The plaque debris collection and analyses were conducted using a paired FilterWire device incubated in patient-derived blood ex vivo to control for proteins in circulating blood that might have otherwise adhered to the device during its deployment. This enabled identification of a highly selected list of proteins that can be specifically attributed to liberation from the plaque, after plaque disruption, and do not represent circulating plasma proteins.

We identified and validated LBP as a novel, plaquederived biomarker of coronary atherosclerotic plaques. LBP is a secretory class 1 acute phase protein, previously associated with the response to bacterial sepsis. Various pathogen associated molecular patterns bind to LBP, and these complexes interact with Toll Like Receptor 4 (TLR4) associated pathways [21]. There is emerging literature indicating a potential role for LBP as a biomarker in atherosclerotic disease. Serum LBP has recently been shown to correlate with prevalent CAD [22] and predicted both all-cause and cardiovascular mortality [23].

Given the role of inflammatory activation by TLR4 in atherogenesis [24], our finding of LBP in human coronary artery plaques in vivo, and plaque-derived systemic release of LBP are novel findings that justify further consideration of LBP as a pathogenic factor in coronary artery disease. Indeed, release of LBP from disrupted plaque could drive the observed increase in MMP9 after plaque disruption, via TLR4 and NFKB signalling. The acute LBP elevation observed in this cohort was followed by significant subsequent increase in systemic MMP9 as described in LXR/RXR canonical pathway activation.

In addition, our workflow also identified other plasma proteins which were acutely elevated after plaque disruption that have plausible biological associations. For example, plasma Annexin A5 has previously been shown to be elevated in patient with acute myocardial infarction [25] and is acutely increased after angioplasty induced AMI [26]. Cytochrome B5 reductase 3 (CYB5R3) is an enzyme involved in the electron transfer pathway in cholesterol synthesis [27] and the elongation and desaturation of fatty acid chains [28]. High levels of circulating monounsaturated fatty acid is recently shown to associate with future cardiovascular events in a large cohorts study [29]. These observations further strengthen the validity of our approach for identifying novel targets.

Further validation by larger cohort(s) will improve the external validity of our findings and to confirm the role of these proteins as novel biomarkers. It will also be important to establish the time course profile of these proteins of plaque disruption, and the range of plasma concentration of these proteins in physiological conditions in large cohorts. 


\section{Conclusion}

We demonstrate a novel approach to discover novel biomarkers of human coronary artery plaque disruption, by combining plaque imaging with proteomic analysis of plaque-derived material, and plasma proteins. We identify the LXR/RXR pathway, and, specifically LBP, as a novel biomarker signature of human coronary artery plaques and plaque disruption. Highly-stratified proteomic analyses of plaque-derived material and plasma can identify new targets of potential biological importance in human coronary artery disease.

\section{Additional files}

\section{Additional file 1. Supplemental method and data.}

Additional file 2: Table S1. Proteins present in the plaque debris. We identified a total of 423 proteins that were derived from the plaque debris, captured by a FilterWire positioned downstream of the disrupted plaque.

Additional file 3. Changes in lipopolysaccharide binding protein after plaque disruption.

\begin{abstract}
Abbreviations
ACS: acute coronary syndrome; CRP: C-reactive protein; CTnl: cardiac troponin I; CyB5R3: cytochrome B5 reductase 3; FW: FilterWire; FW-Control: FilterWire control; MMP9: matrix metalloproteinase 9; LBP: lipopolysaccharide binding protein; LC-MS: liquid chromatography mass spectrometry; LXR/RXR: liver X receptor/retinoic $X$ receptor; OCT: optical coherence tomography; $P C l$ : percu-
\end{abstract} taneous coronary intervention; SA: stable angina; TLR4: toll like receptor 4.

\section{Authors' contributions}

Design and conduct of clinical study: RL, DA, RKK, RPC, CA, KMC. Design and conduct of mass spectrometry study: RL, RF, PDC, AV, KD, BMK. Analysis of data: $\mathrm{RL}, \mathrm{RF}, \mathrm{PDC}, \mathrm{DA}, \mathrm{AV}, \mathrm{KD}, \mathrm{CA}, \mathrm{BMK}, \mathrm{KMC}$. Manuscript drafting: All co-authors. All authors read and approved the final manuscript.

\section{Author details}

${ }^{1}$ Division of Cardiovascular Medicine, University of Oxford, Oxford, UK. ${ }^{2}$ Acute Vascular Imaging Centre, Radcliffe Department of Medicine, University of Oxford, Oxford, UK. ${ }^{3}$ Target Discovery Institute, Nuffield Department of Medicine, University of Oxford, Oxford, UK. ${ }^{4}$ National Institute for Health Research (NIHR) Oxford Biomedical Research Centre, John Radcliffe Hospital, Oxford University Hospitals NHS Foundation Trust, Oxford, UK.

\section{Acknowledgements}

Boston Scientific UK provided Filter Wires for the study.

\section{Competing interests}

The authors declare that they have no competing interests.

\section{Availability of data and materials}

The datasets used and/or analysed during the current study available from the corresponding author on reasonable request.

\section{Ethics approval and consent to participate}

The study was approved by the Oxford Regional Ethics Committee (Ethics Ref: 08/H0603/41) and complies with the Helsinki Declaration. Written informed consent was obtained from every participant.

\section{Funding support}

$R L$ received funding from the Royal Australasian College of Surgeons Scholarship Program and the Academy of Medical Sciences UK. RF and BMK are supported by a Kennedy Trust Award. PDC is supported by a Wellcome Trust Award (105605/Z/14Z) to BMK. CA is a British Heart Foundation Senior Fellow.
The study was funded by National Institute of Health Research (NIHR) Oxford Biomedical Research Centre and supported by the British Heart Foundation Oxford Centre of Research Excellence (RG/13/1/30181) and Chair Award $(\mathrm{CH} / 16 / 1 / 32013)$; to KMC).

\section{Publisher's Note}

Springer Nature remains neutral with regard to jurisdictional claims in published maps and institutional affiliations.

Received: 3 February 2017 Accepted: 9 June 2017

Published online: 19 June 2017

\section{References}

1. Falk E, Nakano M, Bentzon JF, Finn AV, Virmani R. Update on acute coronary syndromes: the pathologists' view. Eur Heart J. 2013;34:719-28.

2. Lindahl B. Are there really biomarkers of vulnerable plaque? Clin Chem. 2011;58(1):151-3.

3. Chen YC, Huang AL, Kyaw TS, Bobik A, Peter K. Atherosclerotic plaque rupture: identifying the straw that breaks the camel's back. Arterioscler Thromb Vasc Biol. 2016;36:e63-72.

4. Higo S, Uematsu M, Yamagishi M, Ishibashi-Ueda H, Awata M, Morozumi T, Ohara T, Nanto S, Nagata S. Elevation of plasma matrix metalloproteinase-9 in the culprit coronary artery in patients with acute myocardial infarction: clinical evidence from distal protection. Circ J. 2005;69:1180-5.

5. Brezinski DA, Nesto RW, Serhan CN. Angioplasty triggers intracoronary leukotrienes and lipoxin A4. Impact of aspirin therapy. Circulation. 1992;86:56-63.

6. Lee T, Yonetsu T, Koura K, Hishikari K, Murai T, Iwai T, Takagi T, lesaka Y, Fujiwara H, Isobe M, Kakuta T. Impact of coronary plaque morphology assessed by optical coherence tomography on cardiac troponin elevation in patients with elective stent implantation. Circ Cardiovasc Interv. 2011;4(4):378-86. doi:10.1161/CIRCINTERVENTIONS.111.962506.

7. Yonetsu T, Kakuta T, Lee T, Takahashi K, Yamamoto G, lesaka Y, Fujiwara H, Isobe M. Impact of plaque morphology on creatine kinase-MB elevation in patients with elective stent implantation. Int J Cardiol. 2011;146:80-5.

8. Ferrante G, Nakano M, Prati F, Niccoli G, Mallus MT, Ramazzotti V, Montone RA, Kolodgie FD, Virmani R, Crea F. High levels of systemic myeloperoxidase are associated with coronary plaque erosion in patients with acute coronary syndromes: a clinicopathological study. Circulation. 2010;122:2505-13.

9. Porto I, Di Vito L, Burzotta F, Niccoli G, Trani C, Leone AM, Biasucci LM, Vergallo R, Limbruno U, Crea F. Predictors of periprocedural (type IVa) myocardial infarction, as assessed by frequency-domain optical coherence tomography. Circ Cardiovasc Interv. 2012;5(89-96):s81-6.

10. Liuzzo G, Buffon A, Biasucci LM, Gallimore JR, Caligiuri G, Vitelli A, Altamura S, Ciliberto G, Rebuzzi AG, Crea F, et al. Enhanced inflammatory response to coronary angioplasty in patients with severe unstable angina. Circulation. 1998;98:2370-6.

11. Porto I, Selvanayagam JB, Van Gaal WJ, Prati F, Cheng A, Channon K, Neubauer S, Banning AP. Plaque volume and occurrence and location of periprocedural myocardial necrosis after percutaneous coronary intervention: insights from delayed-enhancement magnetic resonance imaging, thrombolysis in myocardial infarction myocardial perfusion grade analysis, and intravascular ultrasound. Circulation. 2006;1 14:662-9.

12. Lee R, Antonopoulos AS, Alexopoulou Z, Margaritis M, Kharbanda RK, Choudhury RP, Antoniades C, Channon KM. Artifactual elevation of plasma SCD40L by residual platelets in patients with coronary artery disease. Int J Cardiol. 2013;168:1648-50.

13. Cairns DA, Barrett JH, Billingham LJ, Stanley AJ, Xinarianos G, Field JK, Johnson PJ, Selby PJ, Banks RE. Sample size determination in clinical proteomic profiling experiments using mass spectrometry for class comparison. Proteomics. 2009;9:74-86.

14. Levin $Y$. The role of statistical power analysis in quantitative proteomics. Proteomics. 2011;11:2565-7.

15. Kendziorski C, Irizarry RA, Chen KS, Haag JD, Gould MN. On the utility of pooling biological samples in microarray experiments. Proc Natl Acad Sci USA. 2005;102:4252-7. 
16. Diz AP, Truebano M, Skibinski DO. The consequences of sample pooling in proteomics: an empirical study. Electrophoresis. 2009;30:2967-75.

17. Trudgian DC, Thomas B, McGowan SJ, Kessler BM, Salek M, Acuto O. CPFP: a central proteomics facilities pipeline. Bioinformatics. 2010;26:1131-2.

18. Newby AC. Dual role of matrix metalloproteinases (matrixins) in intimal thickening and atherosclerotic plaque rupture. Physiol Rev. 2005;85:1-31.

19. Galis ZS, Sukhova GK, Lark MW, Libby P. Increased expression of matrix metalloproteinases and matrix degrading activity in vulnerable regions of human atherosclerotic plaques. J Clin Invest. 1994;94:2493-503.

20. Newby AC. Metalloproteinases and vulnerable atherosclerotic plaques. Trends Cardiovasc Med. 2007;17:253-8.

21. Wright SD, Ramos RA, Tobias PS, Ulevitch RJ, Mathison JC. CD14, a receptor for complexes of lipopolysaccharide (LPS) and LPS binding protein. Science. 1990;249:1431-3.

22. Lepper PM, Schumann C, Triantafilou K, Rasche FM, Schuster T, Frank H, Schneider EM, Triantafilou M, von Eynatten M. Association of lipopolysaccharide-binding protein and coronary artery disease in men. J Am Coll Cardiol. 2007;50:25-31.

23. Lepper PM, Kleber ME, Grammer TB, Hoffmann K, Dietz S, Winkelmann BR, Boehm BO, Marz W. Lipopolysaccharide-binding protein (LBP) is associated with total and cardiovascular mortality in individuals with or without stable coronary artery disease-results from the Ludwigshafen Risk and Cardiovascular Health Study (LURIC). Atherosclerosis. 2011;219:291-7.
24. Edfeldt K, Swedenborg J, Hansson GK, Yan ZQ. Expression of toll-like receptors in human atherosclerotic lesions: a possible pathway for plaque activation. Circulation. 2002;105:1158-61.

25. Kaneko N, Matsuda R, Hosoda S, Kajita T, Ohta Y. Measurement of plasma annexin $\mathrm{V}$ by ELISA in the early detection of acute myocardial infarction. Clin Chim Acta. 1996;251:65-80.

26. Peetz D, Hafner G, Blankenberg S, Peivandi AA, Schweigert R, Brunner K, Dahm M, Rupprecht HJ, Mockel M. Annexin V does not represent a diagnostic alternative to myoglobin for early detection of myocardial infarction. Clin Lab. 2002;48:517-23.

27. Porter TD. Electron transfer pathways in cholesterol synthesis. Lipids. 2015;50:927-36.

28. Elahian F, Sepehrizadeh Z, Moghimi B, Mirzaei SA. Human cytochrome b5 reductase: structure, function, and potential applications. Crit Rev Biotechnol. 2014;34:134-43.

29. Wurtz P, Havulinna AS, Soininen P, Tynkkynen T, Prieto-Merino D, Tillin T, Ghorbani A, Artati A, Wang Q, Tiainen M, et al. Metabolite profiling and cardiovascular event risk: a prospective study of 3 population-based cohorts. Circulation. 2015;131:774-85.

\section{Submit your next manuscript to BioMed Central and we will help you at every step:}

- We accept pre-submission inquiries

- Our selector tool helps you to find the most relevant journal

- We provide round the clock customer support

- Convenient online submission

- Thorough peer review

- Inclusion in PubMed and all major indexing services

- Maximum visibility for your research

Submit your manuscript at www.biomedcentral.com/submit 